\title{
Computational Investigation of Varying Plate-Line Geometry and Placement on Wake Vortex Dissipation
}

\author{
C. H. John Wang* \\ Nanyang Technological University, Singapore 639798, Republic of Singapore \\ Dan Zhao \\ University of Canterbury, Christchurch 8140, New Zealand \\ Jörg Schlüter声 \\ Deakin University, Geelong, Victoria 3220, Australia \\ and

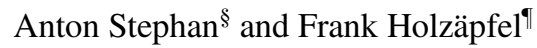 \\ DLR, German Aerospace Center, 82234 Oberpfaffenhofen, Germany
}

\section{DOI: $10.2514 / 1 . C 034346$}

\begin{abstract}
One of the bottlenecks for the recent increase in air traffic density is near the airport, where wake vortices, bounded by the terrain, could easily rebound into the landing corridor and remain there for a long period of time while maintaining their strength. The threat of near-ground wake vortex could be mitigated by the plate-line technology, which places a series of thin plates across the landing corridor before the runway; the technology was shown to significantly reduce the time it takes for wake vortex dissipation. The current investigation aims to better understand factors such as plate dimension and lateral position influences on the generation of secondary vortex structure and ultimately the dissipation rate of a wake vortex pair. It is found that the wake dissipation could be sped up further by optimizing the lateral position of the plate relative to the obstacle-vortex contact location, while the height of the plate could be significantly reduced without noticeably affecting the performance of the obstacle set.
\end{abstract}

\section{Introduction}

A DVANCES in aviation technology and economic development in previously underdeveloped nations around the world in the past few decades has led to a substantial increase in the civil aviation market. The change in air travel market requires civil aviation authorities around the world to look for ways to accommodate the increasingly congested flight infrastructures, both in air and on land. One of the solutions for increasing air traffic density was through the RECAT (wake vortex re-categorisation) program, which would reduce the aircraft separation minima established by the International Civil Aviation Organization (ICAO) with the intention of preventing wake turbulence encounter [1-3]. Because the original wake separation category was developed based on the maximum takeoff mass of the aircraft instead of wake characteristics, a larger aircraft under "heavy" category such as the Boeing 747 following a smaller heavy aircraft such as MD-11 would need to observe the same separation distance as the MD-11 following the 747; this would result in an unnecessary restriction on number of aircraft flying a given path.

The increased density of air traffic en route would mean that hub airports near maximum capacity such as Singapore's Changi International Airport must find a way to efficiently accommodate the incoming traffic while maintaining flight safety. During the development of the Wake Vortex Prediction and Monitoring System, evidence was found that wake behavior near the runway vicinity can

Presented as Paper 2016-4176 at the 34th AIAA Applied Aerodynamics Conference, Washington, D.C., 13-17 June 2016; received 8 January 2017; revision received 6 April 2017; accepted for publication 29 May 2017; published online 31 July 2017. Copyright @ 2017 by Dan Zhao. Published by the American Institute of Aeronautics and Astronautics, Inc., with permission. All requests for copying and permission to reprint should be submitted to CCC at www.copyright.com; employ the ISSN 0021-8669 (print) or 1533-3868 (online) to initiate your request. See also AIAA Rights and Permissions www.aiaa.org/randp.

*Research Fellow, Air Traffic Management Research Institute, 50 Nanyang Avenue.

${ }^{\dagger}$ Associate Professor, Department of Mechanical Engineering, Private Bag 4800; dan.zhao@canterbury.ac.nz. (Corresponding Author).

¥Senior Lecturer, School of Engineering, Locked Bag 20000.

${ }^{\S}$ Research Scientist, Institute of Atmospheric Physics.

${ }^{\ddagger}$ Senior Research Scientist, Institute of Atmospheric Physics. be a bottleneck for reducing flight separation [4]. The study showed that reduced separations were mostly close to the runway threshold. Near ground, the trajectory of an aircraft wake could easily rebound into the path of a following landing aircraft that might not have the altitude required to recover from such an encounter. Additionally, studies regarding the effect of crosswind on wake dissipation near ground $[\underline{5}, \underline{6}]$ have shown that the upwind vortex could hover above the runway and remain a safety hazard for a significantly longer period of time. The need to mitigate wake encounter hazard near airports prompted the development of plate-line obstacles by DLR, German Aerospace Center at Oberpfaffenhofen, Germany [7]. The current study, in collaboration with DLR, aims to increase our understanding of near-ground wake dissipation with obstacles and the further development of the plate line technology for application at airports.

\section{Previous Studies}

The generation of aircraft wake vortices starts due to the pressure imbalance at the wing tip, where air from the high-pressure region below the wing wraps around the wing tip toward the lower-pressure region above the wing. Under certain circumstances, the wake vortex from a larger aircraft could exceed the roll control authority of a trailing aircraft, resulting in aircraft damage and passenger injuries [8]. A good overview of the wake vortex research up until 1990 can be found in the annotated bibliography by Hallock [9]. It included some of the research that formed the basis of ICAO's Wake Turbulence Category, originally published in 1978 under the 11th edition of ICAO Document 4444 [1]. Since then, the exponential growth in civil air travel resulted in greatly increased air traffic density in air and around the airport, resulting in more aircraft traveling closer to the separation minima. Yet even with the implementation of wake turbulence separation between aircrafts, wake turbulence encounters are still being reported, the majority of which occurr during the approach phase of the flight [10]. The increase in air traffic density, the development of Doppler lidar wake measurement system, and the increase in computational capacity in recent years triggered an increase in wake vortex studies, including a number of field tests [11-17], laboratory experiments [17-19], numerical studies [5,20-29], and stability analysis studies $[\underline{23}, \underline{28}, \underline{30}, \underline{31}]$. An up-to-date review dealing 
with dynamics and instabilities of vortex pairs can be found in Leweke et al. [32]. A summary of wake vortex evolution in ground proximity and on the previous works done on the usage of obstacles for wake dissipation is presented next.

As the wake vortices come into the "in ground effect" region, the dissipation of the vortex pair is controlled by the interaction between the vortical flow and the viscous boundary layer near ground. Direct numerical simulation (DNS) [29,32,33] studies at low Reynolds number (up to $R e_{\Gamma}=5,200$ ) have shown that these vortex systems exhibit elliptical instability due to the interaction between the wake vortex and a secondary vortex formed by the vortex-ground interaction (the ground-effect vortex) and would lead to selfamplifying curvature in the secondary vortex. The interaction between the primary and the secondary vortex would lead to the bursting of vortex core upon linking of the two structures [29,34]. Additionally, it was observed that the formation of vortex ring due to Crow instability would not occur unless the initial vortex height is greater than five times the initial vortex separation $b_{0}$. Instead, the vortex deformation due to long-wave instability manifests as vertical half-loops with each end linking with the ground [35]. Large-eddy simulations (LES) [7] conducted at Reynolds number of 23,100 with the addition of crosswind flow showed that the secondary vortex would not detach cleanly from the boundary flow but instead forms hairpinlike structures. However, these flow structures behave much in the same way as the deformed secondary vortex, leading to local bursting of the wake vortex core, where linking of the flow structures occurs.

The LES simulations have showed that the accelerated breakdown of the wake vortex is related to the secondary vortex structure (SVS) wrapping around and linking with the primary vortex core $[7,29]$. This led to the investigation of using externally created $S \bar{V} \bar{S}$ to increase the dissipation rate of the wake vortex in a similar manner, which could be beneficial in cases where accelerated wake vortex dissipation is desirable, such as when atmospheric conditions suppress the formation of SVS or limit the interaction between SVS and the wake vortex.

An early study of wake vortex dissipation using ground-based devices was conducted by Hydronautics under NASA contract [36], which investigated a number of passive and active devices for vortex disruption and containment. However, the experiment was only evaluated based on photographic records, and no qualitative measurements were taken; the experiments were also conducted before our current understanding of SVS's role in wake dissipation was established and did not account for its effect.

The usage of ground-based obstacles for the generation of SVS was first proposed by Stephan et al. [7] in 2013, who conducted LES to study the decay mechanisms of wake vortex behavior near ground and the implementation of ground-based obstacles as artificial enhancement device for wake dissipation. Two different obstacle types were considered in the study: the $0.2 b_{0} \times 0.2 b_{0}(h \times w)$ square cylinder, where $b_{0}$ is the initial wake vortex separation distance, and the $0.1 b_{0} \times 0.2 b_{0}$ "plate line" obstacles. The first obstacle was used for initial LES analysis to visualize SVS creation and the interaction between the SVS and the wake vortex; it was also used in a water towing-tank study conducted in the Wasser Schleppkanal Göttingen (WSG) facility [37]. The plate-line obstacles were developed after considering the construction cost and safety hazard to aircraft in case of runway excursion and to prevent the obstruction of navigation equipment. Although the volume of the obstacle is greatly reduced compared to the square cylinder obstacle, simulations have shown that the plate line provides at least a similar level of wake vortex dissipation. The usage of plate lines for wake encounter mitigation was further studied in flight tests and by simulations using a hybrid Reynolds-averaged Navier-Stokes (RANS)-LES solver that accounts for the aircraft geometry and the termination of wake production upon the touchdown of the aircraft [38-40]. Simulations were also carried out to investigate the effect of crosswind on the performance of the plate lines, which showed that acceleration of vortex decay due to the obstacle plates is still observed under crosswind condition [7]. Optimizations of plate-line parameters was also conducted using a combination of LES solutions and a kriging method algorithm to perform probabilistic multi-element optimization of the plate aspect ratio with fixed height, the interplate separation distance, and the aircraft type [41].

The studies presented in this paper began in late 2014 with the ultimate goal of implementing passive devices to enhance wake dissipation at Singapore's Changi International Airport. To that end, LES studies of different obstacle configurations [42-44] and weather conditions [6] have been conducted using both the open-source OpenFOAM tool set and the in-house JetCode, a branch of Stanford's CDP code. The relevant portion of our obstacle configuration studies is discussed in Sec. III.C.

\section{Simulation Setup}

Numerical simulations in this study are conducted at Nanyang Technological University using the high-memory node on the Nanyang Analytical server hosted by the high-performance computing center. OpenFOAM version 2.2. $\mathrm{x}$ is used in the investigation, with the solver employed being the default incompressible Navier-Stokes equation solver using the pressure implicit with splitting of operator (PISO) algorithm [45]. LES turbulence modeling is used in the study to accurately capture the vortical flow, which could not be done using RANS simulations. On the other hand, running the simulation using DNS for the domain size needed would exceed what the hardware can support. The subgridscale turbulence is resolved using the dynamic Smagorinsky model implemented by Passalacqua, 辛 which obtained the Smagorinsky constant using local cell face averaged values developed by Lilly [46]. Simulations are conducted using dynamic time steps based on a fixed maximum Courant number of 0.8 ; the average Courant number throughout the domain is significantly less than 0.01 . The comparison of simulation data with WSG measurements is presented in Fig. 1. Note that the data for 36.6 million cells are only available until ${t^{*}}^{-}=2.0$.

\section{A. Simulation Domain and Boundary Conditions}

The simulation domain used in this work is based on the testsection dimensions of the water canal from the WSG experiments [7]. The cross-section area of the water canal is $1.1 \times 1.1 \mathrm{~m}$, where the width corresponds to roughly $6 b_{0}$ and the height of the simulation domain is set to $2 b_{0}$ to limit the mesh size and reduce the memory and disk storage requirement; the length of the simulation domain is set to $8 b_{0}$. A schematic of the simulation domain with the baseline obstacle setup used in this study is shown in Fig. 2.

The velocity boundary condition consists of a fully resolved, noslip boundary at the floor, the side walls, and the obstacles; the top wall is set as full-slip wall; the end walls, normal to the axis of the vortex (i.e., the aircraft's direction of travel) employed periodic boundary conditions. The pressure boundary conditions are set to zero-gradient condition, which enforces all pressure gradients normal to the patch to be zero on all surfaces. The subgrid-scale turbulence kinetic energy $k$ on the no-slip walls is set to zero.

The simulation domain is discretized as a structured mesh. Boundary-layer meshes with a sufficient refinement $\left(z^{1}=0.0003 \mathrm{~m}\right.$ on the floor patch) with a growth rate of 1.2 to support fully resolved near-wall flows are implemented for all no-slip surfaces. Calculations show that $\overline{y^{+}}<1.0$ and $y_{\max }^{+}<4.0$ on all no-slip patches throughout the simulation. A small growth rate of 1.01 is implemented on the mesh away from the wall mesh to limit the total mesh size, resulting in a total mesh size of $\sim 12$ million cells for the whole computational domain.

\section{B. Modeling of Wake Vortex}

A simplified wake vortex model is used in the present study based on the assumption that the wake is generated by a wing at low angle of attack with a clean profile. The clean wing assumption allows us to model the vortex detaching from each wing tip using a single vortex

\footnotetext{
${ }^{\dagger}$ Data available online at https://github.com/AlbertoPa/ dynamicSmagorinsky [retrieved April 2014].
} 


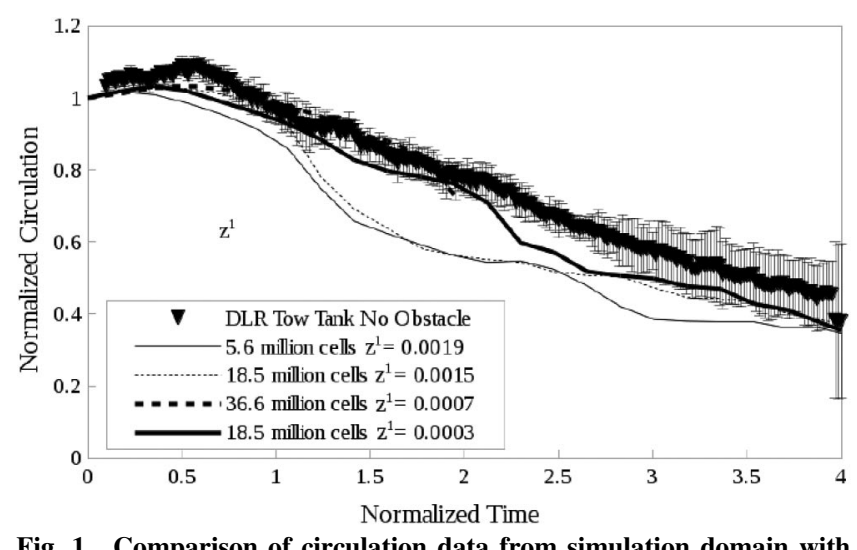

Fig. 1 Comparison of circulation data from simulation domain with various mesh density.

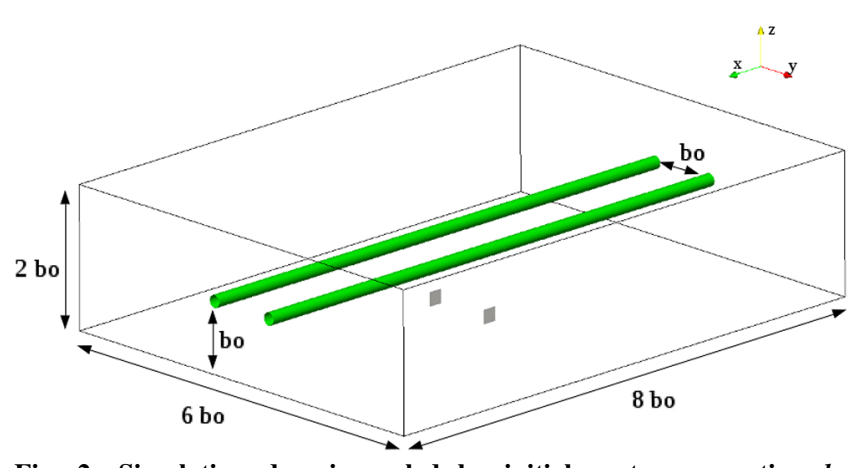

Fig. 2 Simulation domain scaled by initial vortex separation $b_{0}$ (baseline obstacle setup).

structure due to the fast roll up. A wing in high-lift configuration would generate a much noisier wake profile that consists of primary vortex structures, from the wing tip and the flap tips, surrounded by a number of smaller vortices generated by the various protrusions from the wing as well as the wake produced as a result of the higher angle of attack. The initialization of the wake vortex is done by imposing the vortex velocity profile onto the $y-z$ plane all along the $x$ (axial) direction. The resulting vortex structures and their placement within the simulation domain are shown in Fig. 2 .

Although there are several equations describing the velocity profile of wake vortices $[21,24]$, the Lamb-Oseen vortex model is used here to maintain compatibility with previous LES results. The velocity formulation of the Lamb-Oseen vortex is defined as

$$
V_{\theta, 0}(r)=\frac{\Gamma_{0}}{2 \pi r}\left(1-\exp \left(-\frac{r^{2}}{r_{c, 0}^{2}}\right)\right)
$$

It employs the initial values $\Gamma_{0}$ and $r_{c, 0}$ taken from the WSG particle image velocimetry measurements. The initial vortex characteristics from the WSG experiments are listed in Table 1.

The characteristics and circulation decay pattern of the normalized WSG wake vortex pair have been shown to be equivalent to the normalized wake vortex behavior of an Airbus 340 aircraft [7]. However, the Reynolds number used in the WSG study $\left(R e_{\Gamma}=52,000\right)$ and the complementary LES $\left(R e_{\Gamma}=23,100\right)$ are

Table 1 Variables used to initialize the wake vortices based on WSG measurements

\begin{tabular}{ll}
\hline \hline Variable & \multicolumn{1}{c}{ Value } \\
\hline Initial vortex circulation $\Gamma_{0}$ & $0.052 \mathrm{~m}^{2} / \mathrm{s}$ \\
Initial vortex core $r_{c, 0}$ & $0.009 \mathrm{~m}$ \\
Initial vortex separation $b_{0}$ & $0.153 \mathrm{~m}$ \\
Time for normalization $b_{0} / V_{0}\left(t_{0}\right)$ & $2.8258 \mathrm{~s}^{\mathrm{a}}$ \\
\hline \hline
\end{tabular}

${ }^{\mathrm{a} C a l c u l a t e d ~ u s i n g ~} V_{0}=\Gamma_{0} / 2 \pi b_{0}$. both much lower than that which occurrs in practice. The present study is conducted similar to the WSG study, at $R e_{\Gamma}=52,000$.

The initial height of the vortex used in the current study is set to $h_{0}=1.0 b_{0}$, which is different from the $0.5 b_{0}$ used for the cases shown in Fig. 1 and the WSG setup. The initial height is raised to reflect the flight height that has been considered to be more realistic as reported in recent investigations for the adjustment of wake vortex separations by the various civil aviation agencies [47-49].

\section{Ground Obstacles Setup}

The ground obstacle parameters investigated in this work are an extension to the preliminary results as reported in our previous conference papers, which showed LES simulation results using different "shapes" of ground obstacles [42], lateral positioning [43], and aspect ratios, given a constant area of the obstacle plate [4 $\overline{44}$ ]. Selected previous results related to the shape study are included in this section because these influenced the subsequent direction of our investigation on the position and aspect ratio. The results of the later study are presented and discussed in Sec. IV.

The obstacles investigated in the shape study are configured to see if repositioning the plates at an angle would affect the strength and propagation speed of the SVS. A schematic of these obstacles viewed in the top-down direction is displayed in Fig. 3 . The effects of these obstacles on circulation of the modeled wake vortex at predetermined sampling planes are shown in Fig. 4 .

Because of the difference of the ground obstacle shapes, distinct SVS patterns are observed: the SVS generated by baseline and obstacle 1 both consisted of a single $\Omega$ loop structure with counterrotating legs originating from the outer edges of the obstacle; obstacle 2 generates two flow structures, with a weaker $\Omega$ loop at the outer edges and a concentrated airstream at the apex of the chevron; obstacle 3 , modeled as a vortex generator with the aim of preventing wake vortex rebound and prolong contact between the vortex pair and the ground, generates two weaker $\Omega$ loops instead. Examples of the different SVSs generated are shown in Fig. $\underline{5}$.

Although none of the angled obstacle designs are deemed practical due to the limitations of flight safety and navigation, the simulation data do offer some insights for improving the baseline obstacle setup. The differences of the wake dissipation patterns between baseline, obstacle 1 , and obstacle 3 suggest that the $x-z$ projected area of the obstacle plays a more dominant role than the obstacle shape on the strength and propagation speed of the SVS. Furthermore, the dissipation pattern between obstacles 1 and 2 shows that the separation distance between the obstacles affects the timing of initial SVS generation.

The observation on the $x-z$ project area leads us to hypothesize that the wake vortex circulation reduction close to the obstacles is related to the energy being taken out by the interaction between the obstacle and the vortical flow, and thus the obstacle with higher drag would remove more vortex strength. Another way to look at it is that, by maintaining the obstacle $x-z$ project area, which directly affects inviscid drag, the height of the obstacle could be reduced while maintaining a very similar level of wake reduction. This is especially important after discussing with our colleagues at Civil Aviation Authority of Singapore (CAAS) and Changi International Airport, who raised concerns regarding the height of the original obstacles. Further investigation into how to change the obstacle height while maintaining its area to be constant (thus the different aspect ratios) is conducted using the set of ground obstacles presented in Fig. 6 .

On the other hand, the observation on the obstacle separation could lead to further optimization of plate-line formation, which is designed with a $0.45 b_{0}$ lateral separation between ground plates. Our results showed that the obstacle pair with less separation (obstacle 2) began a circulation dissipation earlier. This leads to the hypothesis that the closer placed obstacles would better block the diverging flow between the wake vortex pair, thus delaying the vortices from naturally moving apart while increasing the energy taken out of the vortex system. The placement of the obstacles in the $y$ direction is shown in Fig. 7, where the gray line indicates the initial $y$ position of the wake vortex pair. The $x-z$ profile of all obstacle plates is $0.2 b_{0} \times 0.2 b_{0}$. 


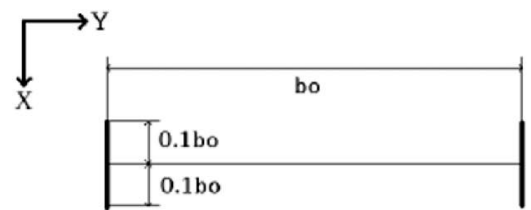

a) Baseline: segBaff

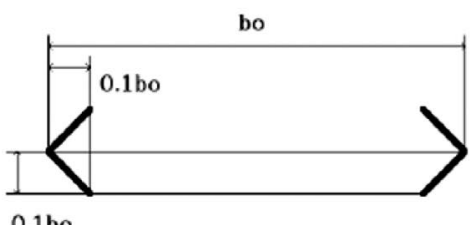

c) Obstacle 2: chvIn

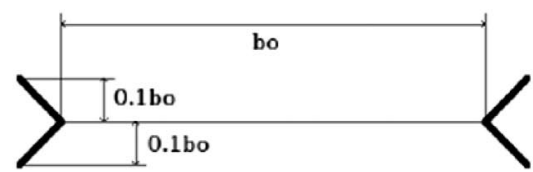

b) Obstacle 1: chvOut

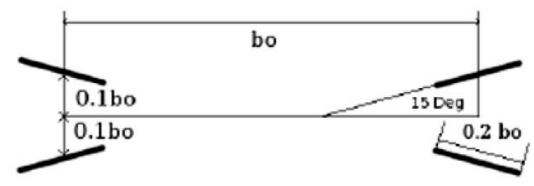

d) Obstacle 3: vortGen

Fig. 3 Top-down view of obstacle dimensions used in the obstacle "shape" study.

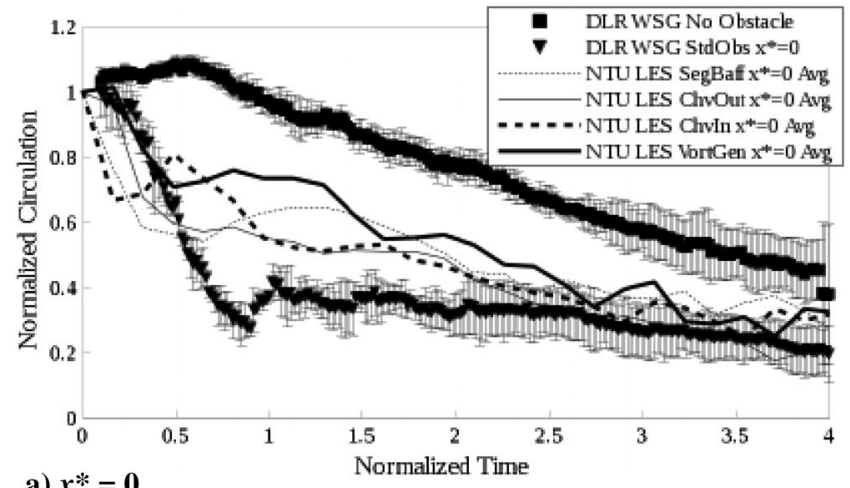

a) $x^{*}=0$

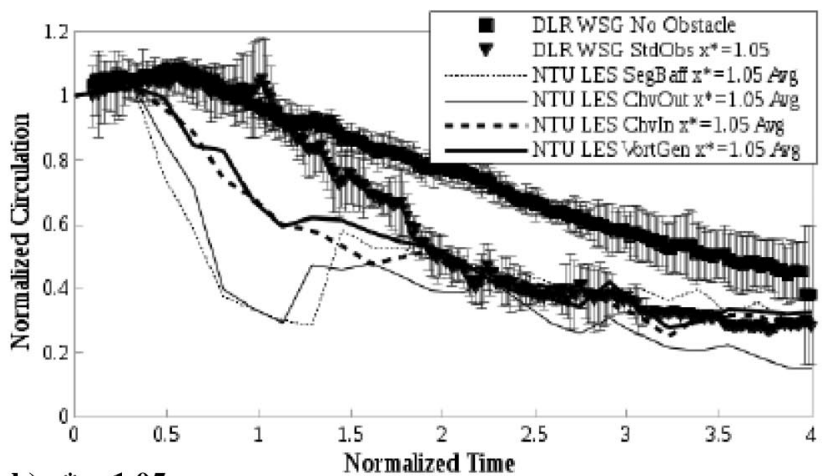

b) $x^{*}=1.05$

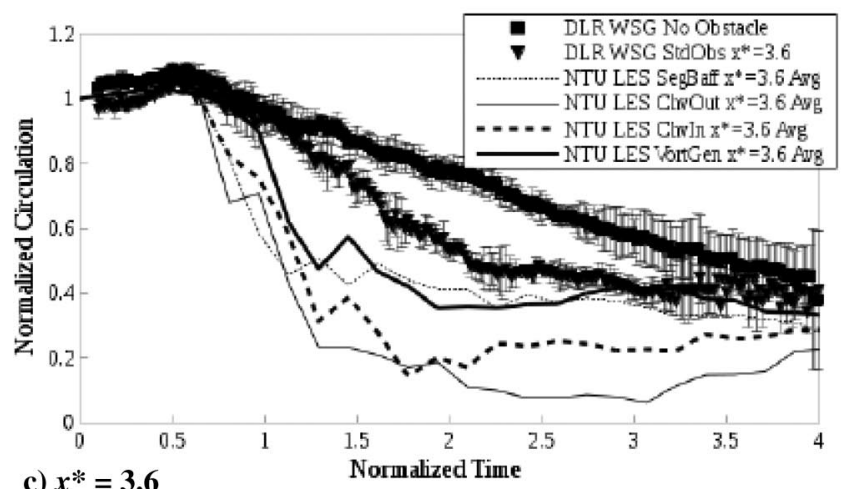

Fig. $4 \quad \Gamma_{\max }^{*}$ [based on Eq. (2)] plot with different obstacle shapes. The experimental data are from the WGS study [17].

An additional set of ground obstacles is tested under the obstacle separation framework that uses multiple plates located at $0.2,0.5$, $0.8 b_{0}$ on each side of the centerline. The separation distance between the plates for the multiplate setup is chosen based on the flow visualization from the previous setups, which showed that the obstacle-generated SVS could extend for up to $0.3 b_{0}$ behind the obstacle before the link between SVS and the obstacle wake is severed. The assumption for this setup is that, by placing the obstacle plates with a $0.3 b_{0}$ separation, the SVS generated would be continuous, resulting in a longer interaction time between the SVS and the wake vortex.

\section{Results and Discussion}

The simulation data are logged at $0.5 \mathrm{~s}\left(t^{*}=0.177\right)$ intervals. The circulation of the wake vortex is evaluated by first extracting data at the sample slice locations $\left(x^{*}=0,1.05\right.$, and 3.6, where $x^{*}=0$ is the center point of the domain, and the positions are normalized with $b_{0}$ ) using postprocessing software ParaView; the extracted data are then postprocessed using the in-house GNU-Octave program that tracks the vortex center and calculates $\Gamma_{\max }$. The center point for each of the wake vortices is tracked by finding the minimum pressure location within a predefined search radius of the previously known vortex center point, whereas the circulation $\Gamma_{\max }$ is calculated using

$$
\Gamma_{\max }=\max _{r}\{\Gamma(r)\}
$$

where the value of $r$ is evaluated at $t^{*}=0$ and coincides with the scaled equivalent of a circulation within $15 \mathrm{~m}$ from the vortex center, $\Gamma_{15}$. It should be noted that, although the value for

$$
\Gamma_{5-15}=0.1 \int_{5}^{15} \Gamma(r) \mathrm{d} r
$$

is commonly used for evaluation of wake vortex hazard, the data from the WSG experiment are only available in $\Gamma_{\max }$. The resulting circulation plots from the different obstacle setups are depicted in Figs. $8-11$.

The influence of the difference in ground obstacle height is especially apparent in Fig. 8a, appearing as cascading dropoff of the circulation curve between $\overline{t^{*}}=0.2 \sim 0.8$ starting from the tallest (lowest aspect ratio) to the shortest (highest aspect ratio). However, the SVS created by the taller obstacles that produced the initial circulation reduction does not appear to be connected to the groundeffect vortex (i.e., the vortex created by the flow separation between wake vortex and ground) and hence is not continuously energized by the ground-effect vortex as described in Stephan et al. [7].

Because of the limited lifespan of these initial SVS, the primary vortex flow straddling the low-circulation region created by the obstacle right above $x^{*}=0$ would be quickly accelerated by the vortical flow on either side due to viscous effects. The large initial reduction in circulation could also be caused by the taller obstacle coming into contact with the wake vortex. The reacceleration of primary vortex segments over $x^{*}=0$ can be seen as the circulation recovery at $t^{*} \sim 0.7$ in Fig. 8a. However, once the subsequent SVS 


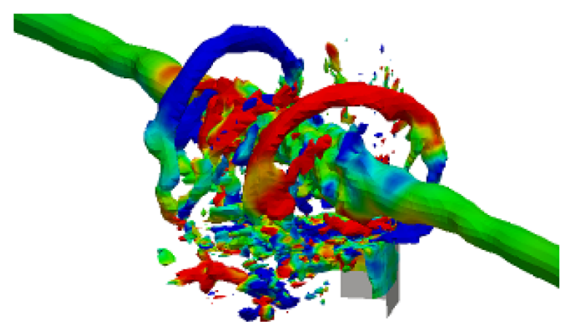

a) Obstacle 1, $\|\omega *\|=170$

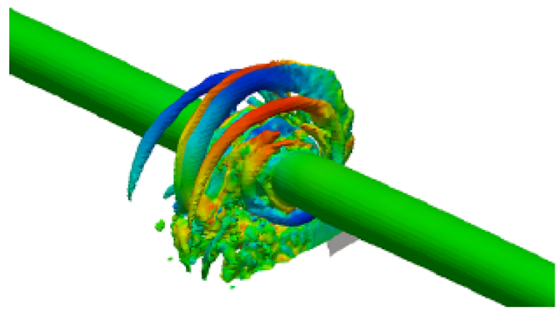

b) Obstacle 3, $\left\|\omega^{*}\right\|=70$

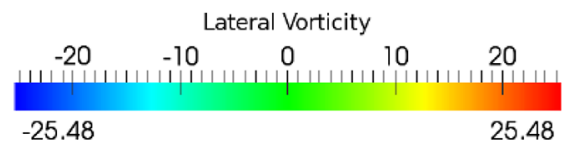

Fig. 5 Isosurface plot showing the SVS generated by different obstacle shapes at $t^{*}=0.354$.

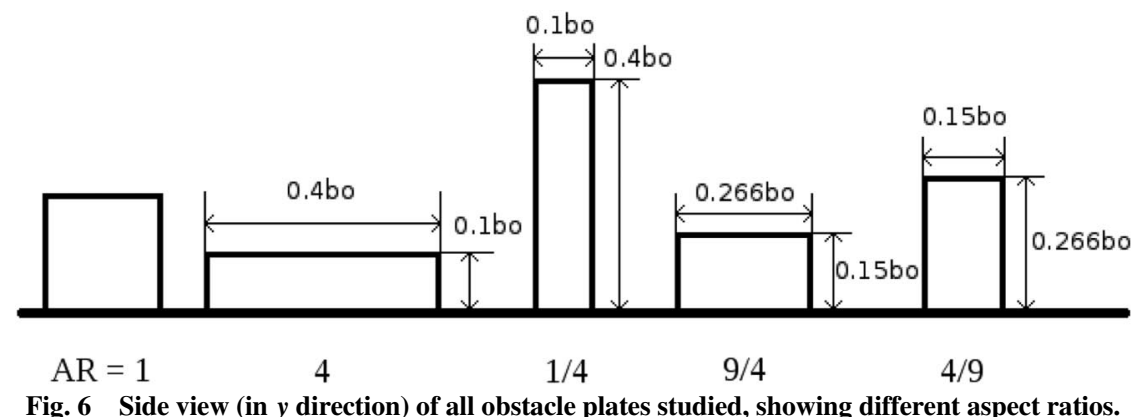

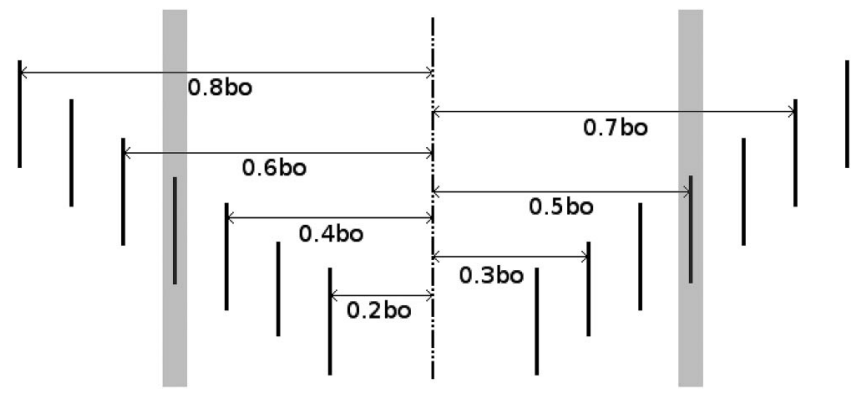

Fig. 7 Obstacle setups used in the separation study.

creation generated by the obstacle is connected to the ground-effect vortex, a more permanent circulation reduction occurs; these SVS would quickly reduce the circulation to a comparable level as all other obstacles by $t^{*} \sim 1.75$.

The influence of the short-lived initial SVS and the subsequent recovery can also be seen farther away from the ground obstacle at $x^{*}=1.05$ (Fig. 8b). The circulation curve of the wake vortex evolution past $t^{*} \equiv 2.0$, however, is still dominated by the groundeffect vortex. On the other hand, the temporary circulation surge from the $A R=1 / 4$ and $R=4 / 9$ cases between $t^{*}=1.5 \sim 2.2$ at $x^{*}=$ 3.6 (Fig. 8 b) is most likely due to the flow collision at the periodic boundary from the remnant of the initial SVS.

The circulation plots from the ground obstacles with three high aspect ratios are shown in Fig. 9. Again, the sequence of the initial dropoff of the circulation curve is related to the height of the obstacle. Even with the delayed creation of SVS comparing to the taller obstacles, the connection of SVS with ground-effect vortex and the subsequent accelerated decay of the primary vortex still occurred. Once the SVS drags the ground-effect vortex around the primary vortex at $t^{*} \sim 2.0$, however, the circulation curve shows similar trends regardless of the obstacles used. This observation echoes the assumption we made that the obstacle with lower profile can be used while the area of the obstacle remains constant.

On the other hand, the lateral separation distance between the obstacle plates appears to have very little impact on the circulation curves for the cases presented in Fig. 10, whereas significant difference can be seen between the baseline case and all other cases presented in Fig. 11. The difference between the two sets of data is likely due to the trajectory of the wake vortex. As a counter-rotating vortex pair descends toward the ground, the flowfield resulting from the ground effect pushes the vortices away from each other and away from the $y$ centerline of the simulation domain. The obstacle pair with the least separation distance is closest to the centerline and thus has the least influence on the wake vortex due to the vortex path. As the lateral separation between the obstacle pair increases past the initial vortex separation distance $b_{0}$, the effect of obstacles on the reduction in circulation becomes a lot more apparent. The more pronounced the circulation reduction is likely due to the proximity of the obstacle plate to the vortex instead of the separation distance between the plates, as previously assumed.

Unlike the cases shown in Fig. 10a, the circulation curve in most cases displayed in Fig. 11a also shows a smoother transition from the obstacle SVS-induced wake vortex decay and the ground-effect vortex powered decay due to the proximity of their position of origin in the lateral direction. The difference in transition can be seen, for example, between the baseline case and the $0.6 b_{0}$ case: the circulation curve for the baseline case reaches a plateau at $t^{*} \sim 1.0$ followed by a kink at $t^{*}=1.5$ that indicates the beginning of decay due to the ground-effect vortex; the same pattern is not seen in the $0.6 b_{0}$ case, showing a smooth line. A more noticeable circulation recovery occurs at $x^{*}=0$ in the $0.8 b_{0}$ case that is not seen in all other cases, yet this recovery is not seen in the other sample slices at $x^{*}=1.05$ and $x^{*}=3.6$, which points to a localized event where the flow feature does not propagate outward; visualizing the flowfield would be needed to further analyze this phenomenon.

Stephan et al. [7] showed that plate lines with a plate size of $0.1 b_{0} \times 0.2 b_{0}$ offered a similar circulation reduction as a square cylindrical obstacle with a cross-sectional area of $0.2 b_{0} \times 0.2 b_{0}$, whereas our previous simulations showed that the baseline plate setup showed lower initial wake dissipation due to SVS compared to the square cylindrical obstacle. As such, the circulation curve of the multiplate setup with a plate separation of $0.3 b_{0}$ meets our expectation compared to the baseline setup. However, the multiplate setup is not expected to show a lower level of dissipation compared to the $0.8 b_{0}$ case because the outermost plates are located at $0.8 b_{0}$ from 


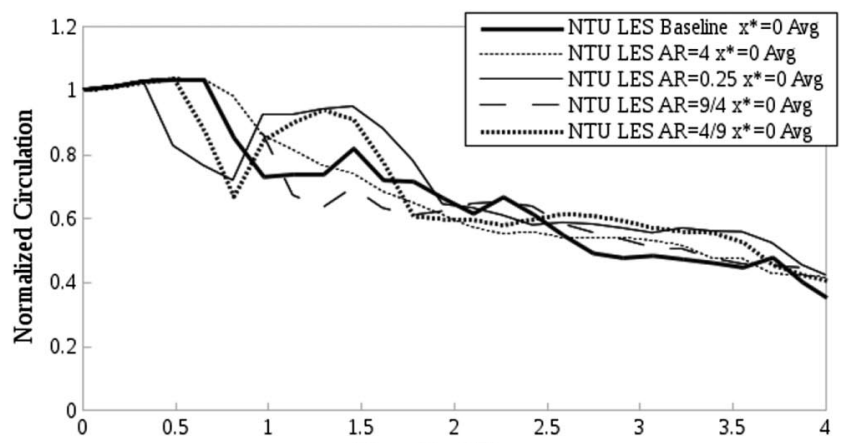

a) $x^{*}=0$

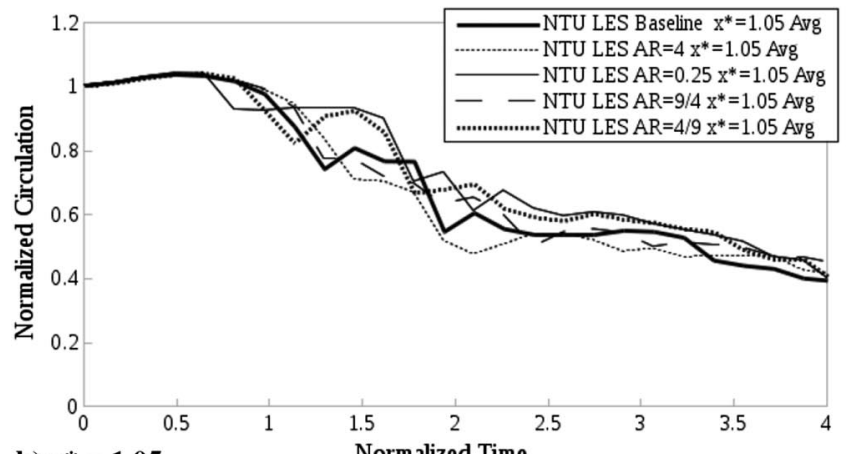

b) $x^{*}=\mathbf{1 . 0 5}$

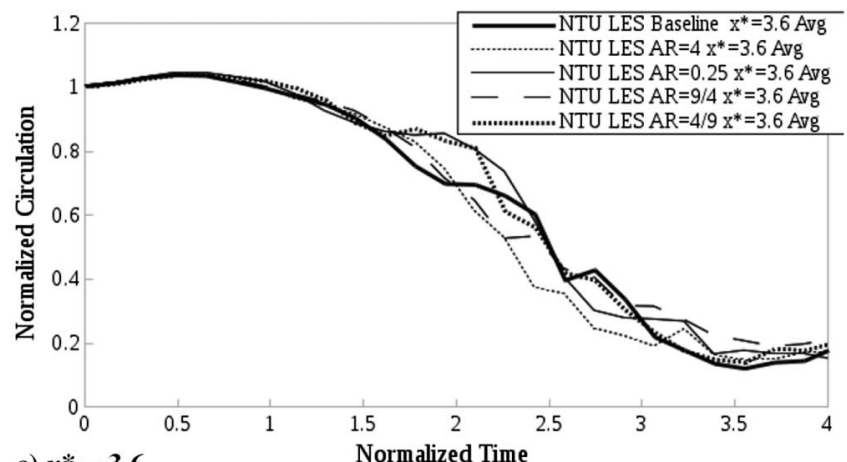

c) $x^{*}=3.6$

Fig. 8 Circulation plots of ground obstacles with a corresponding aspect ratio setup from Fig. $\underline{6}$.

the $y$ centerline in both cases. One explanation for the reduction of the obstacle effectiveness is that instead of extending and adding strength to the SVS created by the preceding plate, the SVS generated by multiple plates would interfere with each other and lower the effectiveness of the setup. This hypothesis could explain the finding of Stephan et al. [41] that ground plates with an interplate separation distances of $0.45 \overline{b_{0}}$ offered the best performance while increasing or reducing that distance both reduces the effectiveness of the obstacle setup. For the plate separation of less than $0.45 b_{0}$, the SVS created from the plate closest to the primary vortex would connect to the ground-effect vortex but fail to sustain the connection as the SVS created from the next plate supersedes it; this would result in a local disruption of the ground-effect vortex, thus reducing its ability to increase wake vortex dissipation directly above the obstacles. On the other hand, the obstacle plates located too far apart would behave in a similar fashion as the $0.2 b_{0}$ case in this study, where the plates are located too far away from the vortex trajectory to have a direct impact with the generation of SVS.

Although the circulation data recorded at the three sample slices are helpful in gauging the relative performance between the different obstacle setups, a better understanding of the interaction between the obstacle-generated SVS, the ground-effect vortex, and the aircraft wake vortex can only be seen using flow visualization. The postprocessing of the simulation data and the flow visualization are

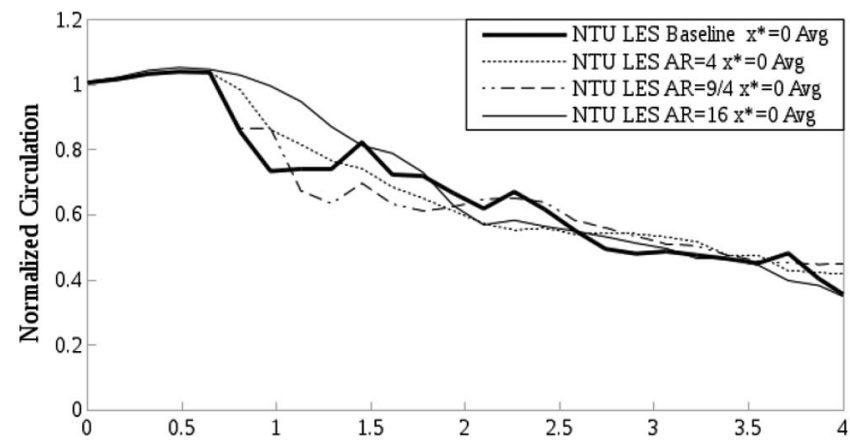

a) $x^{*}=0$

Normalized Time

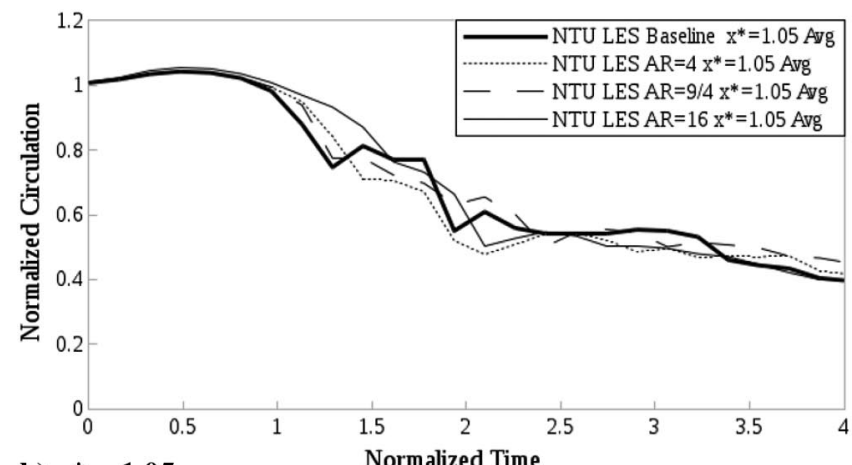

b) $x * 1.05$

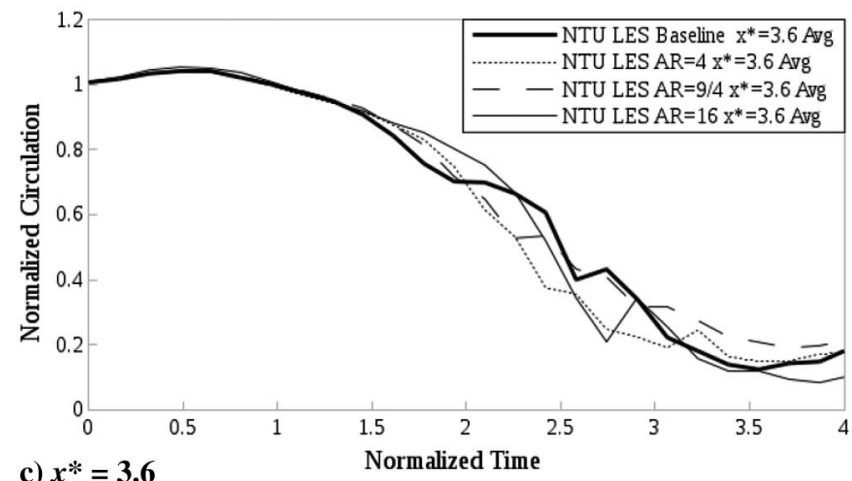

Fig. 9 Circulation plots at three $x^{*}$ locations along the vortex axis for the three high-aspect-ratio obstacle setups comparing to the baseline setup from Fig. 6 .

carried out using the open source postprocessor ParaView- plotting the isosurface of $\left\|\omega^{*}\right\|=79$, where $\omega^{*}=\omega t_{0}$. The color contours on the isosurface are based on lateral vorticity. These visualizations are presented in Figs. 12-15.

Figure 12 demonstrates the influence of the obstacle height on the progress of SVS generation at $t^{*}=0.483$. The difference in the SVS generation is in good agreement with our earlier observation on the obstacle height, as seen in Fig. 8a. Figure 13 highlights the differences between the flowfield generated by different plate aspect ratios using the $A R=4$ and $A R=1 / 4$ cases.

Although the SVS of the $A R=4$ obstacle has yet to complete its wrap around at $t^{*}=0.805$, the SVS from $A R=1 / 4$ has already interwoven into the primary vortex structure and began to spread the instability outward along the vortex axis. It is not until the connection between the SVS and the ground-effect vortex is made that the distinct flow structure could be observed as shown in Fig. 13f. The difference in formation of the SVS would explain the difference of the circulation curves observed in Fig. 8a and is further illustrated in the zoomed-in isosurface plot of the SVS from the aforementioned obstacles in Fig. 14. 


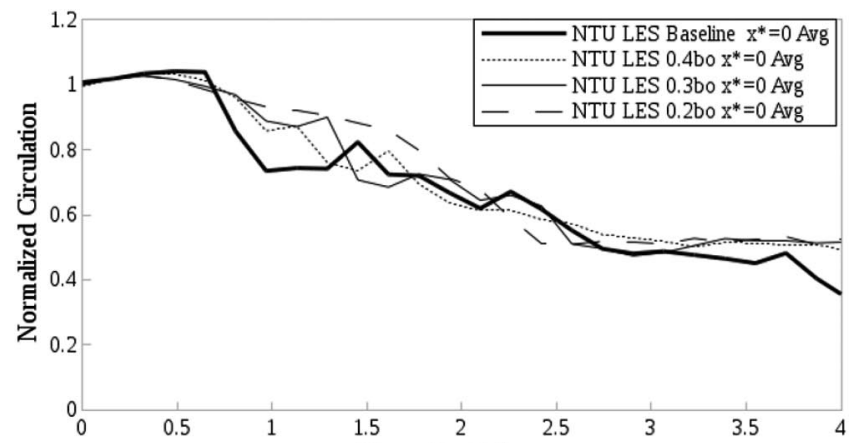

a) $x * 0$

Normalized Time
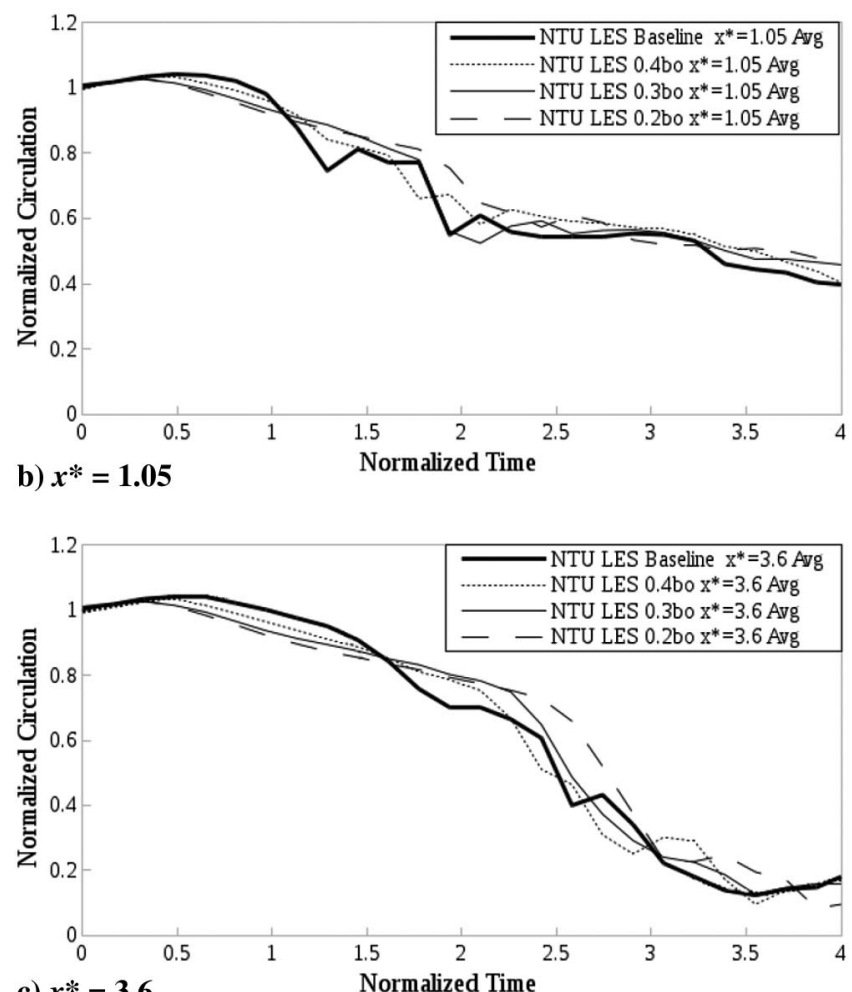

c) $x^{*}=3.6$

Fig. 10 Circulation plots at three $x^{*}$ locations along the vortex axis for obstacle setup (from Fig. 7) located between the initial vortex $y$ position and the wake vortex centerline.

The obstacle-generated SVS can be seen in Fig. 14a to detach from the obstacle's wake while maintaining a distinct flow structure apart from the contact point with the wake vortex. The same observation is not achieved on the taller obstacle. Although the stronger obstaclegenerated SVS from the obstacles with a larger aspect ratio initially reduces the circulation by a larger amount comparing to the ones with a smaller aspect ratio, the advantage is leveled off once the groundeffect vortex contributes to the vortex decay.

For cases where the obstacle plates are placed at different separation distances, we are more interested in the overall structure and the shape of the SVS, as shown in Fig. 15. The arrow in Fig. 15b indicates the deformation caused by the obstacle-generated SVS, whereas the circle indicates the position of the ground-effect vortex. The disconnect between the obstacle-generated SVS and the groundeffect vortex in the circulation plots in Fig. 10a can be seen in the $0.3 b_{0}$ case shown in Fig. $15 \mathrm{c}$. In the case of $0.2 b_{0}$, the obstaclegenerated SVS is too weak to show up in the isosurface plot, but its influence can still be seen with the deformation of the ground-effect vortex. The SVS visualization shows that the difference in circulation reduction between the plate separation setups is related to the connection of the obstacle-generated SVS with the ground-effect vortex.

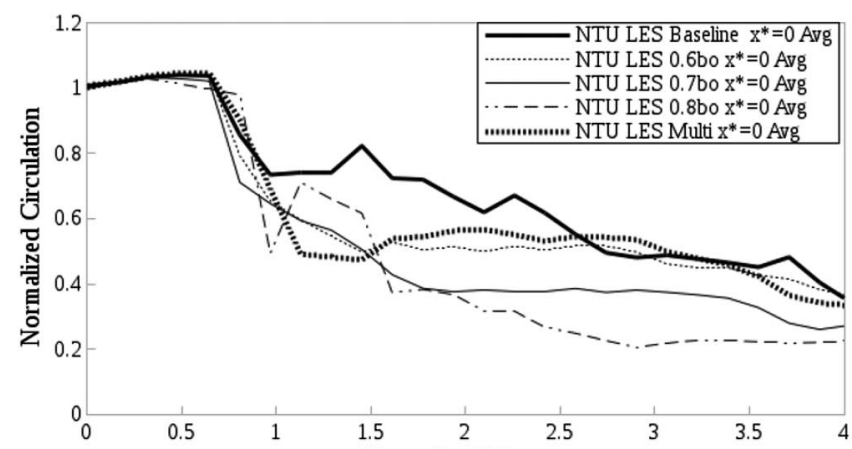

a) $x^{*}=\mathbf{0}$

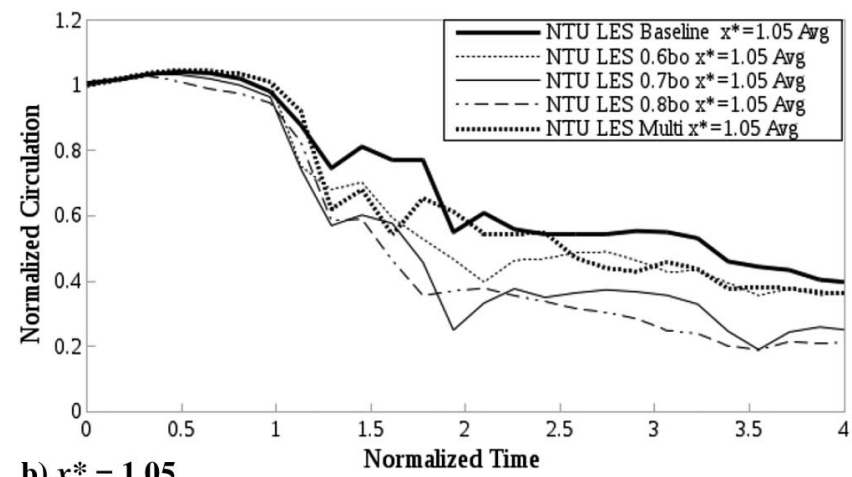

b) $x * 1.05$

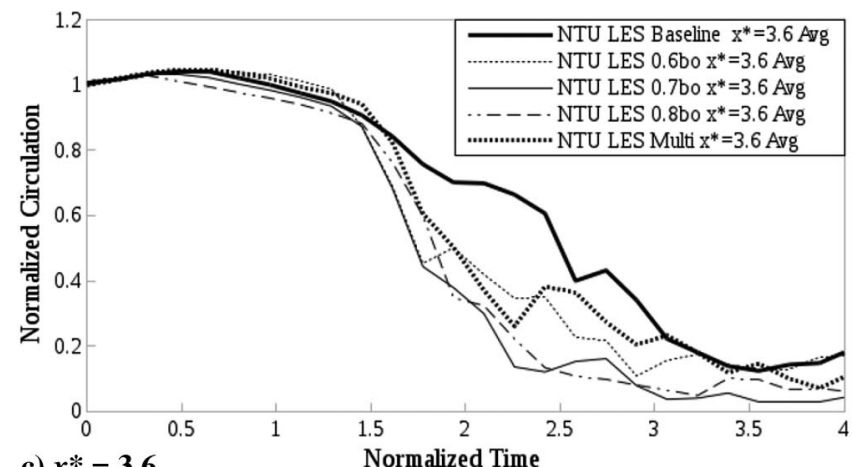

c) $x^{*}=3.6 \quad$ Normalized Time

Fig. 11 Circulation plots at three $x^{*}$ locations along the vortex axis for obstacle setup (from Fig. 7) located beyond the initial wake vortex $y$ position.

The visualization of the $0.8 b_{0}$ case also offers an explanation for the unexpected circulation recovery shown in Fig. 11a. The obstacle plate can be seen protruding into the SVS flowfield circulating the primary vortex structure in Fig. $15 \mathrm{~g}$, thus reducing the integrity and the effectiveness of the SVS flow structure until the vortical flow can be restored by the energy from the ground-effect vortex.

One of the more surprising observations from Fig. 15 is the difference in the diameter of the isosurface tube representing the primary vortex structure. To better understand the reason for this discrepancy, color contours of the vorticity field are taken from the $x^{*}=0$ sample slice and shown in Fig. 16 .

The contour plots show that the primary vortex structure retains its circular shape better in the $0.2 b_{0}$ case, which experiences less flow disruption from SVS but shows a more diffused vorticity profile.

Although quantifying the circulation with $\Gamma_{\max }$ is necessary in the present work because the initial data comparison is done with the WSG data, the typical reported circulation values from the studies on aircraft wake vortex are based on the average of circulation over $5-15 \mathrm{~m}$ around the vortex core, or $\Gamma_{5-15}$. The parameter $\Gamma_{5-15}$ is chosen for comparison because the value derived from simulations shows a better agreement with LIDAR measurements for the initial vortex; the parameter is computed with the equation given as follows: 


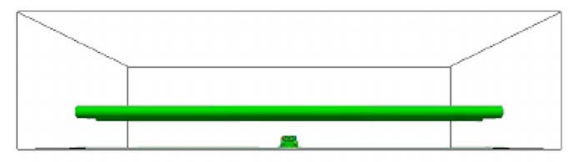

a) Baseline: $A R=1$

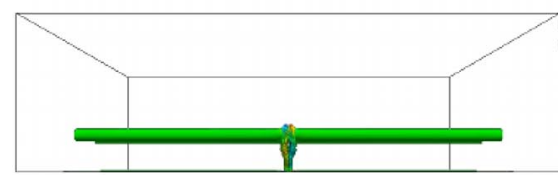

c) $A R=1 / 4$

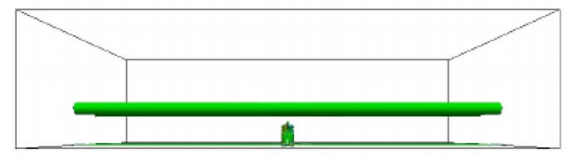

e) $A R=4 / 9$

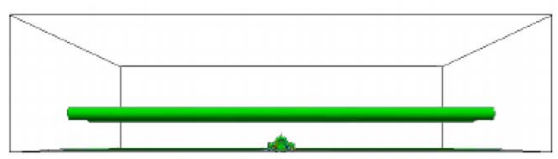

b) $A R=4$

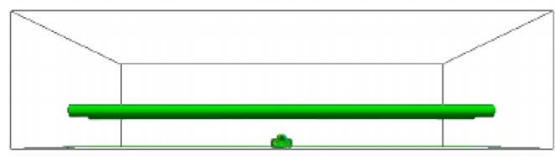

d) $A R=9 / 4$

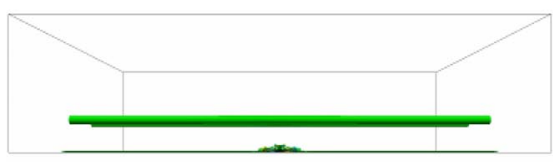

f) $A R=16$

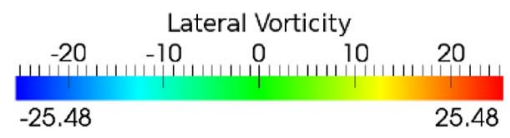

Fig. 12 Visualization at $t^{*}=0.483$, showing isosurface for $\left\|\omega^{*}\right\|=79$ and the onset of secondary vortex structure.

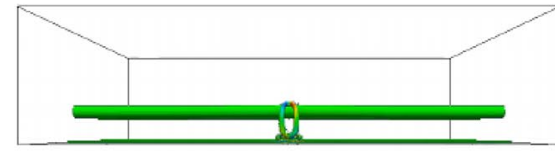

a) $A R=4, t^{*}=0.805$

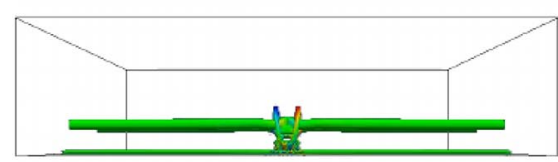

c) $A R=4, t^{*}=0.966$

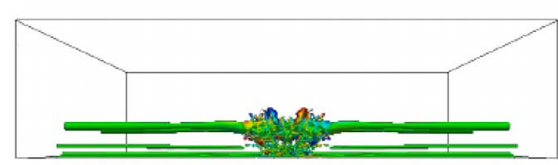

e) $A R=4, t^{*}=1.288$

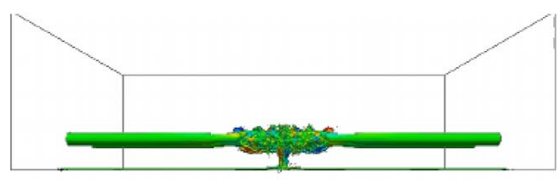

b) $A R=1 / 4, t^{*}=0.805$

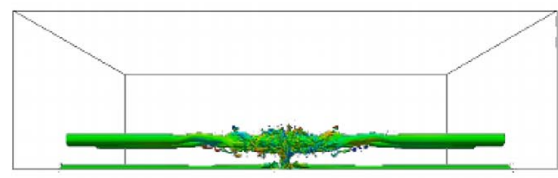

d) $A R=1 / 4, t^{*}=0.966$

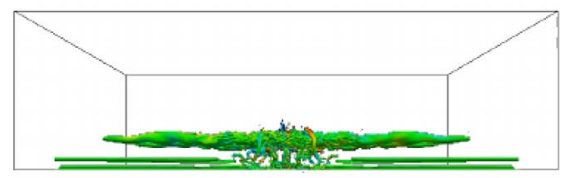

f) $A R=1 / 4, t^{*}=1.288$

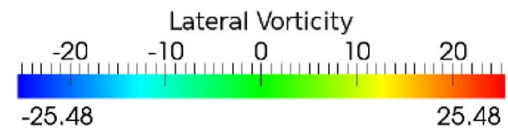

Fig. 13 Visualization with isosurface for $\left\|\omega^{*}\right\|=79$ and the separation in ground-boundary layer for obstacle $A R=4$ and obstacle $A R=1 / 4$.

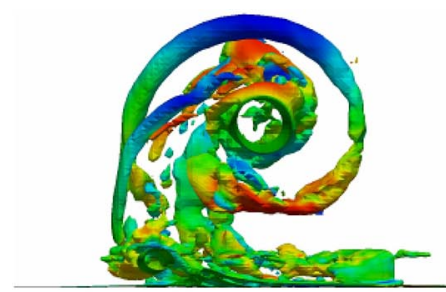

a) $A R=4, t^{*}=1.229$

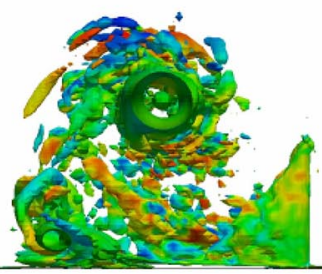

b) $A R=1 / 4, t^{*}=1.229$

$$
\begin{aligned}
& \text { Lateral Vorticity } \\
& -20 \quad-10 \quad 0 \quad 10,20 \\
& -25.48
\end{aligned}
$$

Fig. 14 Isosurface visualization with $\left\|\omega^{*}\right\|=79$ of SVS generated by the obstacles. View in the axial direction. 


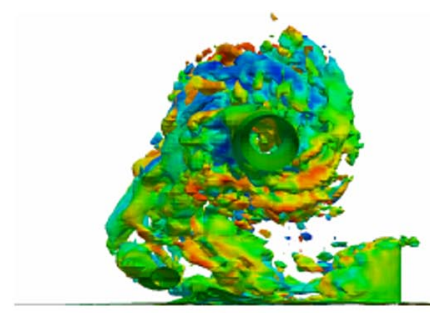

a) Baseline

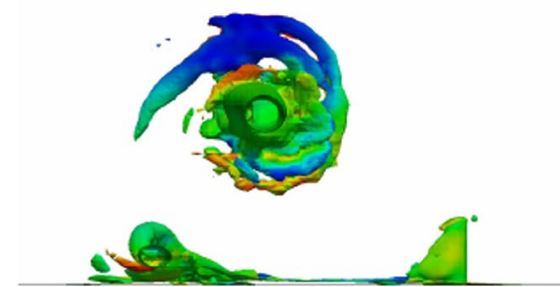

c) $0.3 b_{0}$

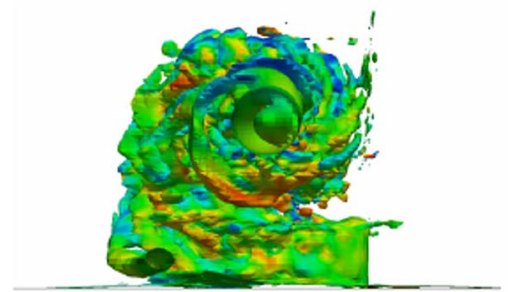

e) $0.6 b_{0}$

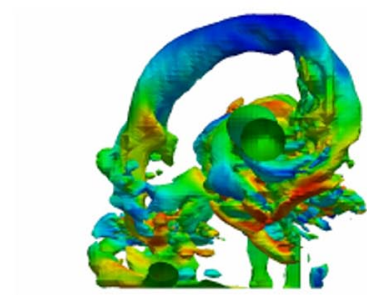

g) $0.8 b_{0}$

\section{0}

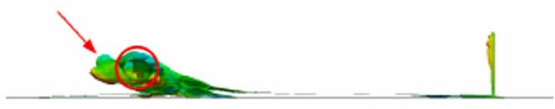

b) $0.2 b_{0}$

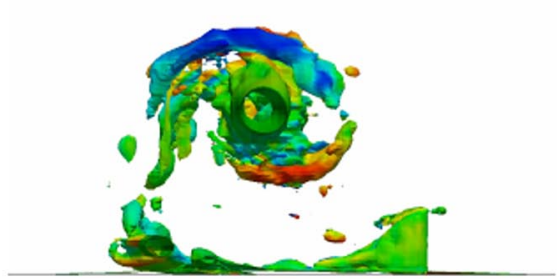

d) $0.4 b_{0}$

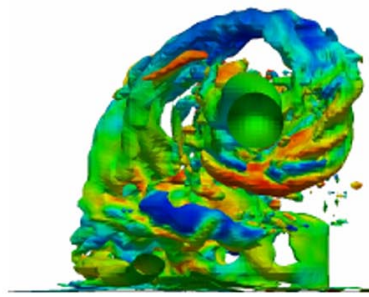

f) $0.7 b_{0}$

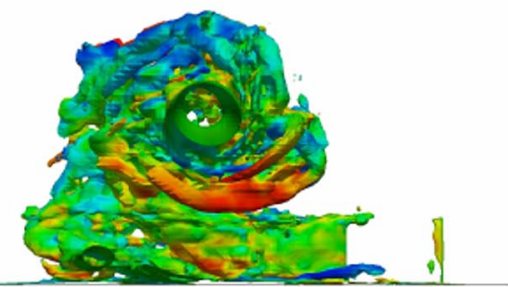

h) Multi Plates

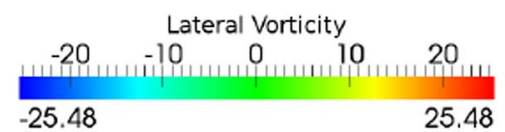

Fig. 15 Isosurface visualization with $\left\|\omega^{*}\right\|=79$ of SVS generated by the obstacles at $t^{*}=1.129$.

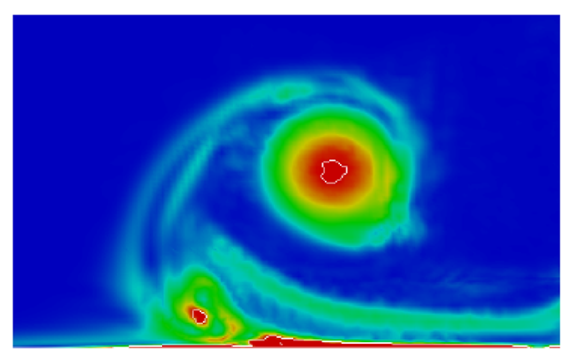

a) $0.2 b_{0}$

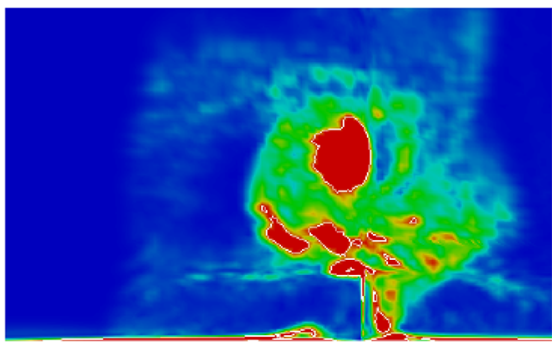

b) $0.6 b_{0}$

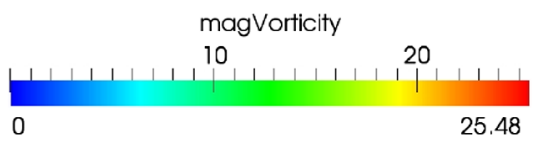

Fig. 16 Color contour of the wake vortex sampled from $x^{*}=0$ at $t^{*}=1.129$ with $\left\|\omega^{*}\right\|=79$ contour line in white.

$$
\Gamma_{5-15}=\frac{1}{10} \int_{5 \mathrm{~m}}^{15 \mathrm{~m}} \Gamma(r) \mathrm{d} r
$$

The circulation distribution plots are constructed by first identifying the vortex center for each of the primary vortices at sample $y-z$ slices with constant $x$ intervals, followed by calculating the $\Gamma_{5-15}$ values for each of the primary vortices at these sample locations and subsequently averaging the two values for port and starboard vortices. For the structured mesh setup, the sampling could also be done at all $x$ positions. The resulting circulation over time data for each of the $x$ positions is then used to construct the pseudocolor contour plots. The vertical axis is the normalized time $t^{*}=t / t_{0}$; the 


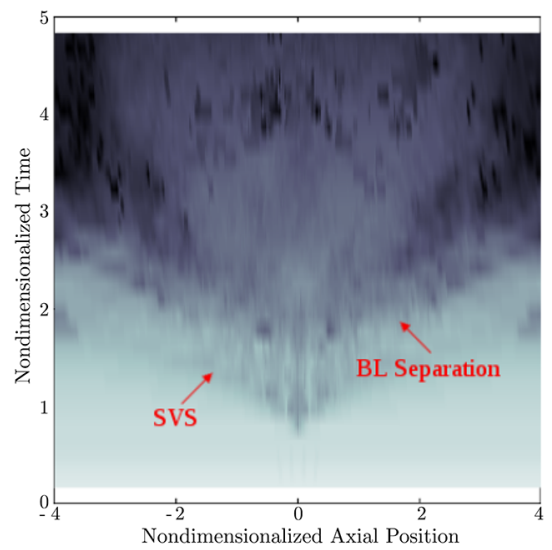

a) Baseline $\left(0.5 b_{0}, A R=1\right)$

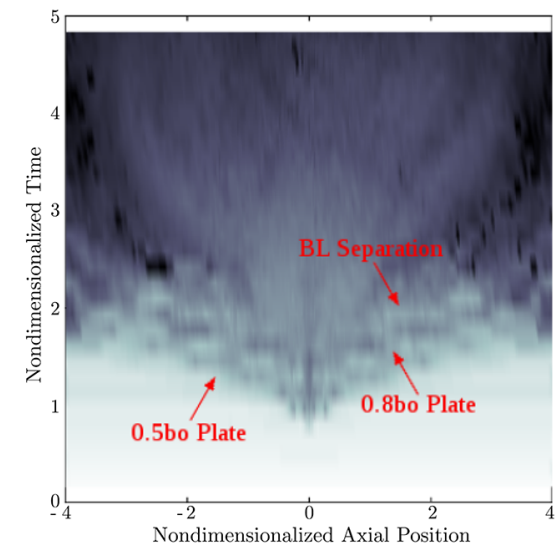

b) $0.2,0.5,0.8 b_{0}, A R=1$

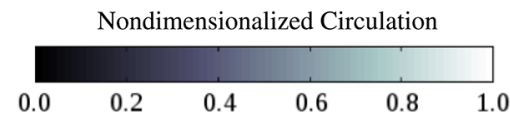

Fig. 17 Circulation distribution with respect to normalized time and normalized axial position.

horizontal axis is the normalized $x$ position $x / b_{0}$, with $x^{*}=0$ being the location of the obstacle; and the color scale is the normalized 5-15 m circulation $\Gamma^{*}=\Gamma / \Gamma_{0}$, where darker colors corresponds to weaker circulation. The domain-wide circulation plots are presented in Fig. 17.

The pseudocolor plots confirm earlier observations that aircraft wake dissipation under the influence of ground obstacles consists of two stages; the first stage is the local decay of the wake vortex due to the interaction between the primary vortex and the obstaclegenerated SVS, and the second stage is the earlier occurrence of the ground-effect vortex decay comparing to the case without ground obstacles, which is induced by the obstacle-generated SVS, and the subsequent propagation of vortex decay along the primary vortex axis. The start of each stage can be seen in Fig. 17 as a darkened line on the pseudocolor plots, indicated by the arrows, as the passing SVS deformed the wake vortex structure. Note that, although the effect of $0.2 b_{0}$ plate on the circulation reduction is not apparent in Fig. $17 \mathrm{~b}$, the early detachment of ground-effect vortex could still be observed in the case where only the $0.2 b_{0}$ plates are installed. The propagation speed of the SVS can also be calculated by the $\Delta t / \Delta x$ slope in the pseudocolor plots as well as the changes in SVS propagation over time.

The multiple-plate case appears to produce a weaker initial reduction through SVS while also showing a delayed onset of the domain-wide vortex decay compared to the plate pairs. This is most likely due to the weaker obstacle-generated SVS because the vortex tangential velocity reduced in magnitude as the flow passes through successive ground plates, creating a weaker SVS behind the obstacle plate each time. The existence of multiple obstacle-generated SVSs also reduces the energy collected from the ground-effect vortex through successive connection and reconnection to the SVS generated by each of the plates. This supports the earlier observation through the isosurface visualization (see Fig. 15) and suggests that these multiple plates should be positioned far enough apart from each other such that the SVS created would not interfere with each other.

Finally, the effect of the periodic boundary conditions in the flight direction can be seen in the contour plots as the darkened region with the reversed slope originating from the $x^{*}= \pm 4$ locations. The results show that the circulation reduction due to flow collision at the domain boundaries could occurs as early as $t^{*}=1.5$, depending on the propagation speed of the initial obstacle-generated SVS.

\section{Conclusions}

Three-dimensional large-eddy simulation studies at $R e_{\Gamma}=$ 52,000 for the purpose of optimizing ground-based obstacle plates for accelerating aircraft wake vortex dissipation are carried out on
Nanyang Technological University's high-performance computing facility using OpenFOAM solvers. The present work considers two hypotheses raised in the authors' previous studies; the first is that the effectiveness of the ground-based plates depends on its area, such that the aspect ratio of these plates can be adjusted while retaining the same level of wake vortex dissipation, and the second is that the separation distance between the parallel plates has some influence on the level of dissipation that could be achieved, although the exact relationship was not quantified in our previous studies. The current study confirms the first hypothesis, showing that, with a constant plate area, the aspect ratio of the plates does not have significant influence on the long-term level of wake vortex dissipation.

On the other hand, the current study shows that the obstacle plates located at more than 0.5 times the initial vortex separations $b_{0}$ from the extended runway centerline ( $y$ centerline for the simulation domain) are associated with a significantly faster dissipation compared to those located within that distance to the centerline. The fully spatial-temporal circulation analysis suggests that this difference may be related to the proximity of the plate to the location where the flow separation of the ground-boundary flow occurred (hence the creation of the ground-effect vortex) in the case without any obstacles. As the SVS extends close enough to the separation location for the "clean" case (without plates), it would induce an earlier separation at the vorticity layer at the ground, resulting in the linking of the obstacle-generated SVS and the ground-effect vortex, leading to an acceleration in decay. The present study also suggests that, in cases where a series of plates are employed, such as in the plate-line setup, having two successive plates too close together would cause an interference between SVSs generated by the plates, ultimately leading to reduced effectiveness of the ground obstacles. This finding is consistent with the results of a plate-line optimization study [41].

The findings from the present study suggest that the obstacle height could be further reduced to achieve similar dissipation performance, which could allow the obstacles to be placed closer to the runway threshold if regulations allow. By placing the obstacle plates closer to the runway threshold, it could be possible to prevent vortex reconnection or the connection of vortices with the ground, thus forming relatively stable half-loops. The findings also suggest that, for setups where crosswind transport of the vortices is not a concern, a single pair of obstacle plates could be sufficient to induce enough wake vortex dissipation within the ground-effect region to avoid the hazard of wake vortex encounter under the current regulations. To substantiate this expectation, a study with a plate pair and a series of weak crosswinds would need to be conducted. 


\section{Acknowledgments}

This work is supported by the Air Traffic Management Research Institute (ATMRI) and DLR, German Aerospace Center under grant number M4061216.056 and University of Canterbury, New Zealand. This financial support is gratefully acknowledged. We would like to thank Thomas Gerz for his feedback and support. The provision of the towing tank data by Reinhard Geisler and Robert Konrath of the Institute of Aerodynamics and Flow Technology, DLR, in Göttingen is greatly acknowledged.

\section{References}

[1] "Procedures for Air Navigation Service: Air Traffic Management," International Civil Aviation Organization, Montreal, Canada, 2007.

[2] "Aircraft Type Designators," International Civil Aviation Organization Rept. 8643, Montreal, Canada, 1996.

[3] "RECAT-EU European Wake Turbulence Categorisation and Separation Minima on Approach and Departure," Eurocontrol, Brussels, Belgium, 2015.

[4] Holzäpfel, F., Dengler, K., Gerz, T., and Schwarz, C., "Prediction of Dynamic Pairwise Wake Vortex Separations for Approach and Landing," 3rd AIAA Atmospheric and Space Environments Conference, AIAA Paper 2011-3037, June 2011.

[5] Holzäpfel, F., Tchipev, N., and Stephan, A., "Wind Impact on Single Vortices and Counter-Rotating Vortex Pairs in Ground Proximity," Flow, Turbulence and Combustion, Vol. 97, No. 3, 2016, pp. 829-848. doi:10.1007/s10494-016-9729-2

[6] Paramasivam, S., Zhao, D., Skote, M., and Schlüter, J., "Detailed Study of Effects of Crosswind and Turbulence Intensity on Aircraft WakeVortex in Ground Proximity," 34th AIAA Applied Aerodynamics Conf., AIAA Paper 2016-4184, 2016.

[7] Stephan, A., Holzäpfel, F., and Misaka, T., "Aircraft Wake-Vortex Decay in Ground Proximity-Physical Mechanisms and Artificial Enhancement," Journal of Aircraft, Vol. 50, No. 4, 2013, pp. 1250-1260. doi: $10.2514 / 1 . C 032179$

[8] "Advisory Circular 90-23G: Aircraft Wake Turbulence," Federal Aviation Administration, Washington, D.C., 2014.

[9] Hallock, J., "Aircraft Wake Vortices: An Annotated Bibliography,” U.S. Department of Transportation, Tech. Rept. DOT-FAA-RD-90-30, 1991.

[10] Veillette, P., "Data Showed That U.S. Wake-Turbulence Accidents Are Most Frequent at Low Altitude and During Approach and Landing," Flight Safety Digest, Vol. 21, Nos. 3-4, March-April 2002, pp. 1-47.

[11] Campbell, S., Dasey, T., Freehart, R., Heinrichs, R., Matthews, M., and Perras, G., "1995 Wake Vortex Testing Program at Memphis, TN," 34th Aerospace Sciences Meeting and Exhibit, AIAA Paper 1996-0399, Jan. 1996.

[12] Campbell, S., Dasey, T., Freehart, R., Heinrichs, R., Matthews, M., Perras, G., and Rowe, G., "Wake Vortex Field Measurements Program at Memphis, Tennessee-Data Guide," NASA, CR-201690, 1997.

[13] Nguyen, D., "Wake Vortex JFK2 Final Report: May 26-June 6, 1997 JFK2 Deployment: Wake Vortex Measurements at John F. Kennedy International Airport for a $2.02 \mathrm{~mm}$ Pulsed Coherent Lidar, a $10.6 \mathrm{~mm}$ Continuous Wave Lidar, and a Ground Wind Vortex Sensing System," Research Triangle Inst., TR RTI/4903-032-03S, North Carolina, 1998.

[14] Nguyen, D., and Britt, C., "Wake Vortex Lidar, NASA Lidar 1997 Dallas/Fort Worth Field Measurements and Data Guide," Research Triangle Inst., TR RTI/4903-032-04, North Carolina, 1998.

[15] Burnham, D., "B-747 Vortex Alleviation Flight Tests: Ground-Based Sensor Measurement," U.S. Dept. of Transportation, TR DOT-TSCFAA-81-19, Washington, D.C., 1982.

[16] Burnham, D., and Hallock, J., "Measurements of Wake Vortices Interacting with the Ground," Journal of Aircraft, Vol. 42, No. 5, 2005, pp. $1179-1187$. doi:10.2514/1.10929

[17] Stephan, A., Holzäpfel, F., Misaka, T., Geisler, R., and Konrath, K., "Enhancement of Aircraft Wake Vortex Decay in Ground Proximity: Experiment Versus Simulation," CEAS Aeronautical Journal, Vol. 5, No. 2, 2014, pp. 109-125. doi:10.1007/s13272-013-0094-8

[18] Allen, A., and Breitsamter, C., "Experimental Investigation of CounterRotating Four Vortex Aircraft Wake," Aerospace Science and Technology, Vol. 13, Nos. 2-3, 2009, pp. 114-129. doi:10.1016/j.ast.2008.05.004

[19] Breitsamter, C., "Wake Vortex Characteristics of Transport Aircraft," Progress in Aerospace Sciences, Vol. 47, No. 2, 2011, pp. 89-134. doi:10.1016/j.paerosci.2010.09.002
[20] Proctor, F., "Numerical Simulation of Wake Vortices Measured During the Idaho Falls and Memphis Field Programs," 14th Applied Aerodynamics Conference, AIAA Paper 1996-2496, 1996.

[21] Proctor, F., Ahmad, N., Duparcmeur, F., and Jacob, D., "Review of Idealized Aircraft Wake Vortex Models," 52nd Aerospace Science Meeting, AIAA Paper 2014-0927, 2014.

[22] Sarpkaya, T., "New Model for Vortex Decay in the Atmosphere," Journal of Aircraft, Vol. 37, No. 1, 2000, pp. 53-61. doi:10.2514/2.2561

[23] Holzäpfel, F., Gerz, T., and Baumann, R., "The Turbulent Decay of Trailing Vortex Pairs in Stably Stratified Environments," Aerospace Science and Technology, Vol. 5, No. 2, 2001, pp. 95-108. doi:10.1016/S1270-9638(00)01090-7

[24] Gerz, T., Holzäpfel, F., and Darracq, D., "Commercial Aircraft Wake Vortices," Progress in Aerospace Sciences, Vol. 38, No. 3, 2002, pp. 181-208. doi:10.1016/S0376-0421(02)00004-0

[25] Holzäpfel, F., Hofbauer, T., Darracq, D., Moet, H., Garnier, F., and Gago, C. F., "Analysis of Wake Vortex Decay Mechanisms in the Atmosphere," Aerospace Science and Technology, Vol. 7, No. 4, 2003, pp. 263-275. doi:10.1016/S1270-9638(03)00026-9

[26] Holzäpfel, F., and Steen, M., "Aircraft Wake-Vortex Evolution near Ground Proximity: Analysis and Parametrization," AIAA Journal, Vol. 45, No. 1, 2007, pp. 218-227. doi: $10.2514 / 1.23917$

[27] Hennemann, I., and Holzäpfel, F., "Large-Eddy Simulation of Aircraft Wake Vortex Deformation and Topology," Proceedings of the Institution of Mechanical Engineers, Part G: Journal of Aerospace Engineering, Vol. 225, No. 12, 2011, pp. 1336-1350. doi: $10.1177 / 0954410011402257$

[28] Meunier, P., Dizès, S. L., and Leweke, T., "Physics of Vortex Merging," Comptes Rendus Physique, Vol. 6, Nos. 4-5, 2005, pp. 431-450. doi:10.1016/j.crhy.2005.06.003

[29] Winckelmans, G., Cocle, R., Dufresne, L., Capart, R., Bricteux, L., Daeninck, G., Lonfils, T., Duponcheel, M., Desenfans, O., and Georges, L., "Direct Numerical Simulation and Large Eddy Simulation of Wake Vortices: Goring from Laboratory Conditions to Flight Conditions," Proceedings of the European Conference on Computational Fluid Dynamics ECCOMAS CFD, Paper 2006-09-08, TU Delft, Egmond aan Zee, The Netherlands, 2006.

[30] Stuart, T., Mao, X., and Gan, L., "Transient Growth Associated with Secondary Vortices in Ground Vortex Interactions," AIAA Journal, Vol. 54, No. 6, 2016, pp. 1901-1906. doi:10.2514/1.J054484

[31] Jacquin, L., Fabre, D., Sipp, D., Theofilis, V., and Vollmers, H., "Instability and Unsteadiness of Aircraft Wake Vortices," Aerospace Science and Technology, Vol. 7, No. 8, 2003, pp. 577-593. doi:10.1016/j.ast.2003.06.001

[32] Leweke, T., Le Dizés, S., and Williamson, C., "Dynamics and Instabilities of Vortex Pairs," Annual Review of Fluid Mechanics, Vol. 48, 2016, pp. 507-541. doi:10.1146/annurev-fluid-122414-034558

[33] Corjon, A., and Stoessel, A., "Three-Dimensional Instability of Wake Vortices near the Ground," 28th Fluid Dynamics Conference, AIAA Paper 1997-1782, 1997.

[34] Ortega, J., Bristol, R., and Savaş, O., "Experimental Study of the Instability of Unequal-Strength Counter-Rotating Vortex Pairs," Journal of Fluid Mechanics, Vol. 474, No. 1, Jan. 2003, pp. 35-84. doi: $10.1017 / \mathrm{S} 0022112002002446$

[35] Williamson, C., Leweke, T., Asselin, D., and Harris, D., "Phenomena, Dynamics and Instabilities of Vortex Pairs," Fluid Dynamics Research, Vol. 46, No. 6, 2014, Paper 061425. doi:10.1088/0169-5983/46/6/061425

[36] Kohl, R., "Model Experiments to Evaluate Vortex Dissipation Devices Proposed for Installation on or near Aircraft Runways," NASA CR132365, 1973.

[37] Stephan, A., Holzäpfel, F., and Misaka, T., "Towards Realistic Simulation of Wake-Vortex Evolution During Landing with Flat and Complex Terrain," Proceedings of the International Symposium on Turbulence and Shear Flow Phenomenon, Poitiers, France, 2013.

[38] Holzäpfel, F., Stephan, A., Körner, S., and Misaka, T., "Wake Vortex Evolution During Approach and Landing with and Without Plate Lines," 52nd Aerospace Science Meeting, AIAA Paper 2014-0925, 2014.

[39] Stephan, A., Holzäpfel, F., and Misaka, T., "Hybrid Simulation of Wake-Vortex Evolution During Landing on Flat Terrain and with Plate Line," International Journal of Heat and Fluid Flow, Vol. 49, Oct. 2014, pp. 18-27. doi:10.1016/j.ijheatfluidflow.2014.05.004 
[40] Holzäpfel, F., Stephan, A., Heel, T., and Kroner, S., "Enhanced Wake Vortex Decay in Ground Proximity Triggered by Plate Lines," Aircraft Engineering and Aerospace Technology, Vol. 88, No. 2, 2016, pp. 206-214.

doi:10.1108/AEAT-02-2015-0045

[41] Stephan, A., Schrall, J., and Holzäpfel, F., "Numerical Optimization of Plate Lines Design for Enhanced Wake Vortex Decay," Journal of Aircraft, Vol. 54, No. 3, 2017, pp. 995-1010.

[42] Wang, C. J., Schlüter, J., Zhao, D., Stephan, A., and Holzäpfel, F., "LES Study on the Shape Effect of Ground Obstacles on Wake Vortex Dissipation," Aerospace Science and Technology, Vol. 63, April 2017, pp. $245-258$ doi:10.1016/j.ast.2016.12.032

[43] Wang, C. J., Schlüter, J., Zhao, D., Stephan, A., and Holzäpfel, F., "Effect of Ground Obstacle Separation Distance on Wake Vortex Dissipation," 34th AIAA Applied Aerodynamics Conference, AIAA Paper 2016-4176, 2016.

[44] Wang, C. J., Schlüter, J., Zhao, D., Stephan, A., and Holzäpfel, F., "Effect of Ground Obstacle of Different Aspect Ratio on Wake Vortex
Dissipation," 8th Atmospheric and Space Environments Conference, AIAA Paper 2016-3134, 2016.

[45] Issa, R. I., Gosman, A. D., and Watkins, A. P., "The Computation of Compressible and Incompressible Recirculating Flows by a NonIterative Implicit Scheme," Journal of Computational Physics, Vol. 62, No. 1, 1986, pp. 66-82. doi:10.1016/0021-9991(86)90100-2

[46] Lilly, D. K., "A Proposed Modificaiton of the Germano Subgrid-Scale Closure Method," Physics of Fluid A, Vol. 4, No. 3, 1992, pp. 633-635. doi:10.1063/1.858280

[47] Crouch, J., and Czech, M., "Comparative Wake-Turbulence Assessments and Findings for the B747-8," Proceedings of the 4th WakeNet3-Europe Workshop, RTCA, Inc., Washington, D.C., 2012.

[48] Deluca, D., "One Year of Time-Based Separation at London's Heathrow Airport," Proceedings of WakeNet-USA, NASA, Washington, D.C., Feb. 2016.

[49] "Federal Aviation Administration (FAA) Aircraft Wake Turbulence Re-Categorization (RECAT) Updates," Safety Alert for Operators 14007, Federal Aviation Administration, Washington, D.C., 2014. 